\title{
Effects of nicotine on the thermodynamics of the DPPC phase coexistence region
}

\author{
Ernanni D. Vieira ${ }^{a}$, A. J. Costa-Filho ${ }^{\text {b }}$ Luis. G. M. Basso, ${ }^{\text {b** }}$
}

${ }^{a}$ Laboratório de Física Biológica, Instituto de Física, Universidade Federal de Goiás, Av. Esperança, s/nº. Campus Samambaia 74.690-900 - Goiânia, GO, Brazil

${ }^{b}$ Laboratório de Biofísica Molecular, Departamento de Física, Faculdade de Filosofia, Ciências e Letras de Ribeirão Preto, Universidade de São Paulo, Ribeirão Preto, SP, Brazil

* Corresponding author. Phone/fax: +55 (16) 3373-8094.

E-mail address: luisbasso@usp.br (Luis G. M. Basso). 


\section{ABSTRACT}

Phase separation plays critical roles in several membrane functions, and

2 reduction or disappearance of phase coexistence by action of membrane-interacting

3 molecules have been implicated in membrane function impairment. Here, we applied

4 differential scanning calorimetry, electron paramagnetic resonance (EPR), and non-

5 linear least-squares (NLLS) spectral simulations to study the effects of nicotine, a

6 parasympathomimetic drug, on the two-phase coexistence of dipalmitoyl

7 phosphatidylcholine (DPPC) lipid membrane. The thermodynamic quantities describing

8 the DPPC phase coexistence are temperature dependent, giving rise to non-linear van't

9 Hoff behavior. Our results showed that nicotine preferentially binds to the fluid phase

10 and modifies the enthalpy and entropy changes of the DPPC heat capacity profile, while

11 marginally perturbing the homogeneous gel and fluid phases. An EPR/NLLS/van't Hoff

12 analysis of the DPPC phase coexistence revealed that nicotine significantly modified the

13 temperature dependence of the free energy change of the two-phase equilibrium from a

14 cubic to a parabolic behavior, resulting in an alteration of the thermodynamical driving

15 force and the balance of the non-covalent interactions of the lipids in equilibrium. The 16 thermotropic behavior of the enthalpy, entropy, and heat capacity changes, as 17 determined by EPR, indicated that nicotine modified the relative contributions of 18 hydrogen-bonding, electrostatic interactions, and conformational entropy of the lipids to 19 the thermodynamics of the phase coexistence. The predominantly entropically-driven 20 gel-fluid transition in nicotine-free DPPC changes to a temperature-triggered 21 entropically-driven or enthalpically-driven process in nicotine-bound DPPC. Further 22 applications of this thermodynamic EPR/NLLS/van't Hoff analysis are discussed.

24 Keywords: nicotine, pulmonary surfactant, EPR, DSC, van't Hoff, drug-membrane interactions. 


\section{Introduction}

Biological membranes consist of complex mixtures of proteins, carbohydrates, and a wide variety of lipids with different hydrocarbon chains, polar groups, backbone structures, type of chemical linkages, etc [1]. Because of their complexity, studying biological membranes remains a major challenge from both experimental and theoretical perspectives. Therefore, a common strategy has been to investigate simple model membranes comprised of only one lipid or mixtures of lipids with the purpose of understanding the puzzle of membrane heterogeneity and intermolecular interactions. Calorimetric techniques such as differential thermal analysis (DTA) and differential scanning calorimetry (DSC) have been frequently used to investigate the physicochemical properties of model and biological membranes [2]. Both techniques can provide direct information on the thermodynamics of the systems under study and, indirectly, on the molecular interactions in those systems [2]. DTA and DSC have also been used to study the effects of different additives such as drugs, polymers, peptides, and proteins in the lipid thermal transitions and in the corresponding phase diagrams of model and biological membranes [2-4]. However, the measure of heat flow does not allow for a complete description of the thermotropic processes at a molecular level. Therefore, specific structural alterations related to inter- and/or intramolecular interactions should be addressed using techniques other than DSC or DTA.

Electron paramagnetic resonance (EPR) of spin labels relies on the use of paramagnetic probes, mainly constituted by stable nitroxide radicals, covalently bound to the molecule of interest, and thus acting as "reporter" groups [5]. Spin labeled phospholipids have been widely used to monitor changes in membrane fluidity, packing of the hydrocarbon chains, local polarity surrounding the spin label, and phase transition temperatures, among other parameters, in model and biological membranes [6-9]. The 
1 sensitivity makes EPR one of the methods of choice when one intends to probe the

2 thermotropic behavior of important microscopic parameters such as the local ordering

3 and mobility of phospholipids in lipid bilayer membranes. The ability of EPR to track

4 down alterations in the membrane dynamical structure can be further improved when

5 simulations of the EPR spectra are included in the analyses [10-12]. Particularly, non-

6 linear least-squares (NLLS) simulations are a powerful tool in decomposing

7 multicomponent EPR spectra from spin probes partitioned into different phases that

8 coexist in model and biological membranes [13-16]. If the partition coefficient of the

9 probe is known, the equilibrium constant $K$ between the lipid states of a two-phase

10 membrane is calculated by determining the fraction of the spin-labeled lipids in both

11 states directly from NLLS spectral simulations. Therefore, van't Hoff analyses can be

12 performed upon recording changes in the equilibrium constant triggered by an external

13 stimulus such as temperature. By plotting $\ln K$ versus reciprocal temperature $1 / T$, one

14 can thus spectroscopically obtain important thermodynamic quantities such as the

15 enthalpy change $\left(\Delta H^{0}\right)$, the entropy change $\left(\Delta S^{0}\right)$, the free energy change $\left(\Delta G^{0}\right)$, and the

16 heat capacity change $(\Delta C)$ of the process under investigation, without the need to

17 directly measure heat flows. Therefore, EPR/NLLS in combination with van't Hoff

18 analysis emerge as an effective tool to indirectly extract meaningful thermodynamical

19 information from the coexistence of phases displaying different EPR observables such

20 as motional and/or structural properties in model and biological membranes.

21 We have previously applied this EPR/NLLS approach to study the

22 thermodynamics of the ripple phase of pure dipalmitoyl phosphatidylcholine (DPPC)

23 membranes and the phase coexistence region of a ternary mixture comprised of DPPC,

24 palmitoyl-oleoyl phosphatidylcholine (POPC), and palmitoyl-oleoyl

25 phosphatidylglycerol (POPG), which mimics the pulmonary surfactant lipid matrix [6]. 
1 Van't Hoff analyses yielded non-linear curves, i.e., the dependence of $\ln K$ on $1 / T$ was

2 not linear over the temperature range corresponding to the phase coexistence region of

3 the membranes, implying a temperature dependence of the thermodynamic parameters.

4 Non-linear van't Hoff plots have also been observed in several different biological

5 applications [17-22].

In the present work, we seek to extend this method to study how small molecules such as drugs alter the thermodynamics, or ultimately, the van't Hoff behavior of phase coexistence regions of model membranes. In particular, the drug of choice is nicotine, a

9 natural product found in the nightshade family of plants, which is a potent 10 parasympathomimetic stimulant acting as an agonist at most nicotinic acetylcholine 11 receptors [23]. Although the mechanisms governing pulmonary nicotine absorption have been well studied [24], there is still no research on the effect of nicotine on the non-covalent interactions that stabilize the first barrier of protection of the pulmonary alveoli. The pulmonary surfactant matrix comprises of about $80 \%$ of phospholipids by weight, from which DPPC corresponds to approximately 50-70\% [25]. Additionally, in 16 eukaryotic cell membranes, phosphatidylcholine (PC) and phosphatidylethanolamine (PE) are the major lipid components, responsible for ca. 50 up to $70 \%$ of the lipid matrix in most membranes [26], with DPPC being one of the most abundant and studied PC lipids. Therefore, the lipid of choice in the present work was DPPC given its biological importance. In such a simple model membrane, the effects of foreign molecules, such as drugs [27, 28], peptides [29, 30] and proteins [31, 32] on the physico-chemical properties of the membrane provided a better understanding of the molecular details underlying those interactions.

Given that DPPC is the most abundant lipid in mammalian lung surfactant, we are particularly interested in investigating the effects of nicotine on the structural 
1 dynamics and on the thermodynamics of the two-phase region of DPPC bilayers, thus

2 putatively mimicking its effect on the pulmonary lung surfactant matrix, which displays

3 as main biological function the reduction of the surface tension at the air-liquid [33]. To

4 do so, we used DSC and EPR spin label spectroscopy along with NLLS spectral

5 simulations to investigate the thermotropic behavior of DPPC membranes in the

6 presence of nicotine. Our experimental results show that nicotine profoundly alters the

7 van't Hoff behavior of the phase coexistence region of DPPC membranes and

8 consequently the thermodynamic balance between the non-covalent interactions of the

9 DPPC bilayer.

10

\section{Materials and methods}

\subsection{Reagents}

The phospholipid 1,2-dipalmitoyl-sn-glycero-3-phosphocholine (DPPC) and the spin label 1-palmitoyl-2-stearoyl(16-doxyl)-sn-glycero-3-phosphocholine (16-PCSL) were purchased from Avanti Polar Lipids, Inc. (Alabaster, AL). The (-)-1-methyl-2-(3pyridyl)pyrrolidine was purchased from Sigma-Aldrich Brasil Ltda (Sao Paulo, Brazil).

All reagents were used without further purification.

\subsection{Sample preparation}

Multilamellar vesicles of DPPC for DSC and EPR were prepared as described

17 elsewhere [6]. Briefly, a measured volume of the nicotine stock solution in ethanol was

18 added to DPPC (for DSC) or DPPC/16-PCSL (for EPR) in chloroform at different

19 lipid:drug molar ratios: $50: 1,25: 1,10: 1,5: 1$, or $2: 1$. The organic solvent was vortexed thoroughly, evaporated under a $\mathrm{N}_{2}$ flow, and the resulting lipid film was placed under 
1 vacuum for $12 \mathrm{~h}$. Pure lipid and lipid/nicotine samples were hydrated with a buffer

2 containing $10 \mathrm{mM}$ acetate, borate, phosphate, $\mathrm{pH} 7.4$, sonicated for $10 \mathrm{~min}$ at $45^{\circ} \mathrm{C}$ in a

3 bath-type sonicator, and freeze-thaw cycled ten times prior to the experiments.

\subsection{Differential scanning calorimetry (DSC) experiments}

\subsection{Electron paramagnetic resonance (EPR) measurements} using Microcal Origin software.

DSC measurements were carried out in a VP-DSC MicroCal microcalorimeter (Microcal, Northampton, MA, USA). The thermograms were recorded from 20 to $55{ }^{\circ} \mathrm{C}$ using a heating rate of $18.1{ }^{\circ} \mathrm{C} / \mathrm{h}$, after a 10 -min equilibration period at $20{ }^{\circ} \mathrm{C}$. The concentration of DPPC used was $2 \mathrm{mg} / \mathrm{ml}(2.72 \mathrm{mM})$. Data analysis were performed

EPR experiments were carried out in a Varian E109 spectrometer operating at X-band $(9.4 \mathrm{GHz})$ using the following acquisition conditions: microwave power, 10 $\mathrm{mW}$; field modulation frequency, $100 \mathrm{kHz}$; field modulation amplitude, 0.2-0.5 G depending on the temperature range; sweep width, $160 \mathrm{G}$. The temperature was controlled using an E257-X Varian temperature control unit coupled to the spectrometer (uncertainty about $0.2{ }^{\circ} \mathrm{C}$ ). The samples were transferred to glass capillaries $(1.5 \mathrm{~mm}$ I.D.) and centrifuged at $10,000 \mathrm{rpm}$ for $10 \mathrm{~min}$. The capillaries containing the lipid pellets were then bathed in mineral oil (for thermal stability) inside an EPR quartz tube and placed in the center of the resonant cavity. Prior to measuring the EPR spectra, the sample was thermalized at each temperature for about three minutes. Typically, lipid samples contained $0.5 \mathrm{mg}$ of DPPC and $0.5 \mathrm{~mol} \%$ of 16 -PCSL relative to the lipids.

\subsection{Non-linear least-squares (NLLS) EPR spectral simulations}


The NLSL software package, developed by Freed and co-workers [10], was used

2 for the simulations of the EPR spectra of the 16-PCSL incorporated in the DPPC lipid

3 bilayers. The most commonly used set of parameters in the calculation of the EPR

4 spectrum are: the magnetic parameters, constituted by the $g$-tensor $\left(g_{x x}, g_{y y}, g_{z z}\right)$ and the

5 hyperfine splittings $\left(A_{x x}, A_{y y}, A_{z z}\right)$, the components of the rotational diffusion tensor $\left(R_{x x}\right.$,

$6 R_{y y}, R_{z z}$ ), and the ordering potential $U(\Omega)$, which is written as a series of spherical

7 harmonic functions represented by the coefficients $c_{20}, c_{22}, c_{40}, c_{42}$, and $c_{44}[10,11]$. In

8 addition, in experiments with multilamellar vesicles the nitroxide-labeled lipid is

9 considered microscopically ordered but macroscopically disordered and, therefore, the

10 so-called microscopic order macroscopic disorder (MOMD) model is used $[10,11]$. The

11 rotational diffusion tensor $(R)$ of the spin-labeled lipid is described in terms of the local

12 director of the bilayer, that is, it is calculated around axes parallel $\left(R_{p l l}\right)$ and 13 perpendicular $\left(R_{p r p}\right)$ to the symmetry axis of the lipid acyl chain and the ordering

14 potential, which describes the orienting influence of anisotropic fluids, such as

15 membranes. As reported elsewhere [34], the 16-PCSL incorporated in lipid bilayers

16 generally presents $R_{p l l} \gg R_{p r p}$, and, following a standard procedure [10, 12], we set $R_{p l l}$ $17=10 R_{\text {prp }}$.

\section{Results and Discussion}

Membrane lipids can exist in a variety of organized states when hydrated [35].

Among the many factors that influence membrane polymorphism, temperature has been

21 the most studied. DSC is one of the most extensively used techniques to study the

22 thermotropic phase behavior and phase diagrams of biological model membranes [35].

23 The DSC thermogram exbhits the temperature dependence of the excess heat capacity 24 of the membranes. The lipid acyl chain melting transition of one-component membranes 
1 is assumed to represent a simple two-state endothermic process, from which the

2 transition midpoint temperature $\left(T_{m}\right)$, where the transition is $50 \%$ complete, can be

3 directly measured and the calorimetric enthalpy change of the transition $\left(\Delta H_{\text {cal }}\right)$

4 calculated from the area of the peak. At the $T_{m}$, the change in the Gibbs free energy of

5 the system is zero [36], allowing for the calculation of the entropy change $(\Delta S)$

6 associated with the transition.

Figure 1A shows representative DSC thermograms illustrating the thermotropic

8 phase behavior of fully hydrated multilamellar DPPC vesicles in the absence and

9 presence of nicotine. Pure DPPC displays two endothermic transitions, a low-enthapic

and broad pretransition centered at $T_{p}=34{ }^{\circ} \mathrm{C}$, and a very intense and more cooperative

11 main phase transition, centered at $T_{m}=41^{\circ} \mathrm{C}$. The low-temperature peak arises from the

12 transition of the solid-ordered lamellar gel phase, $L_{\beta}$, to the ripple gel phase, $P_{\beta}$,

13 whereas the high-temperature peak arises from the conversion of $P_{\beta^{\prime}}$ to the fluid, liquid

14 crystalline $L_{\alpha}$ phase. In the $L_{\beta^{\prime}}$ phase, the lipids adopt an all-trans configuration and display a tilt angle of about $30^{\circ}$ with respect to the membrane normal [37], whereas in the $L_{\alpha}$ phase, the lipid chains are mostly disordered and display many trans and gauche

17 isomerizations in their $\mathrm{C}-\mathrm{C}$ bonds. The $P_{\beta^{\prime}}$ phase is characterized by periodic undulations (ripples) on the membrane surface and is likely formed by periodic arrangements of both $L_{\beta^{\prime}}$ and $L_{\alpha}$ lipid domains [37]. The transition temperatures agree well with our previous works [6, 29].

Addition of nicotine perturbs the heat capacity profile of DPPC in a concentration-dependent manner (Figure 1B). The thermograms of the nicotinecontaining DPPC are progressively shifted towards low temperature, suggesting the

24 drug preferentially distributes into the $L_{\alpha}$ phase of the membrane. Similar results were obtained with the antimalarial drug primaquine [27], whereas trans-parinaric acid 
1 caused an opposite effect, indicating a better partition into the gel phase [38]. Nicotine

2 induced slight changes of the chain-melting temperature of DPPC/nicotine membranes

$3\left(\Delta T_{m}<1{ }^{\circ} \mathrm{C}\right)$, even at the highest lipid-to-drug molar ratio $(2: 1)$. This result implies that

4 nicotine does not perturb the bilayer packing in a great extent. Indeed, the cooperativity

5 of the transition, as qualitatively indicated by the inverse of the linewidth at half

6 intensity $\left(\Delta T_{m, 1 / 2}\right)$, is virtually the same for all conditions (Table 1$)$. Rigorously, the

7 cooperative unit is calculated from the ratio of the van't Hoff enthalpy change $\left(\Delta H_{v H}\right)$

8 and $\Delta H_{\text {cal. }}$. The average number of molecules in a cluster that melts cooperatively as a

9 unit in the absence and presence of nicotine does not change considerably, ranging from $10 \quad 275$ to 335 (Table 1$)$.

11 It has been shown that the pretrasition of phosphatidylcholines is very sensitive 12 to the presence of foreign molecules at the membrane/water interface [27, 39]. 13 Interestingly, binding of nicotine does not significantly alter either the $T_{p}$ or the shape of 14 the pretransition curve (Figure 1A, inset). This result suggests neither the structure of the DPPC head group [40] nor the membrane hydration [41, 42] are strongly affected by nicotine adsorption on the bilayer surface.

The most pronounced effects induced by nicotine were observed in the calorimetric enthalpy change of the whole transition, $\Delta H_{c a l}$ (Figure 1B), and in the respective entropy change, $\Delta S$, at the melting temperature $T_{m}$ (Table 1 ). Both $\Delta H_{\text {cal }}$ and

$20 \Delta S$ increase in a dose-dependent manner, but not exceeding $14 \%$ of the value obtained 21 for the empty vesicles. These results indicate that more heat is needed to melt the acyl chains of nicotine-bound DPPC vesicles and more entropy is gained in this process. 
1 dynamical changes taking place during the phase transition, which requires molecule-

2 based methods to complement the DSC information.

EPR has been widely used to investigate the effect of temperature on model membranes [6,27-29,43,44]. Nitroxide-labeled phospholipids, such as n-PCSL $(n=5,7$, $10,12,14$, and 16 , where $\mathrm{n}$ represents the position of the paramagnetic probe along the

6 lipid acyl chain), are used to dissect the structural rearrangements experienced by the 7 lipid matrix due to changes in the environment. Here, we chose the phospholipid 168 PCSL, spin-labeled at the $16^{\text {th }} \mathrm{C}$-atom of the sn- 2 chain, because it has been shown to

9 be very sensitive to detect phase coexistence between lipid micro-domains exhibiting 10 different ordering and dynamics in EPR experiments [6,13]. Non-linear least-squares 11 (NLLS) spectral simulations of the 16-PCSL EPR spectra were carried out to evaluate changes in the order parameter and the rotational diffusion rates of the lipid spin probe in the membrane. NLLS analysis is very effective in distinguishing the relative population of the spin probes between domains/phases with distinct membrane packing and fluidity [14-16, 27-29].

Figure S1 of the Supplementary Material shows the experimental and best-fit EPR spectra of 16-PCSL in hydrated multilamellar vesicles of DPPC in the absence and presence of nicotine at two lipid-to-drug molar ratios: 25:1 and 10:1. The EPR spectra recorded above ca. $40{ }^{\circ} \mathrm{C}$ from all samples displayed three narrow lines characteristic of a fast lipid probe in the disordered, liquid crystalline $L_{\alpha}$ phase of the bilayers. On the other hand, below a certain temperature that varied between 21 and $28{ }^{\circ} \mathrm{C}$ depending on the sample, the 16-PCSL EPR spectra exhibited broad lines, typical of spin probes in slow motion in the solid-ordered, $L_{\beta^{\prime}}$ phase of the DPPC membranes. In the temperature interval ranging from 28.3 to $40.1{ }^{\circ} \mathrm{C}$ for pure DPPC (Fig. S1A), 26.3 to $40.6{ }^{\circ} \mathrm{C}$ for the 
1 DPPC/nicotine 10:1 molar ratio (Fig. S1E), the spectra presented overlap of broad and

2 narrow components. A single spectral component could not be satisfactorily fitted to the

3 EPR spectra recorded in those regions. The introduction of a second spectral population

4 to the fitting process significantly improved the agreement between the experimental

5 and theoretical curves (Figures S1B, S1D, and S1F). This result indicates phase

6 polymorphism in those regions, that is, coexistence of micro-domains exhibiting

7 different structural organization and membrane fluidity [6,27]. Indeed, it has been

8 shown that the ripple phase of fully hydrated phosphatidylcholine lipid bilayers displays

9 properties of both $L_{\beta^{\prime}}$ and $L_{\alpha}$ phases [45,46]. Interestingly, the appearance of the second

10 spectral component takes place at a temperature lower than the onset temperature of the

11 pretransition observed by DSC (about $29-30{ }^{\circ} \mathrm{C}$, inset of Figure 1A), which may reflect

12 changes in the 16-PCSL conformation due likely to microscopic lipid rearrangements of

13 the surrounding lipids. This result shows the sensitivity of a molecule-based technique

14 such as EPR in detecting different local structural changes before macroscopic

15 endothermic events monitored by calorimetric techniques take place. The higher the

16 nicotine concentration, the lower is the EPR-detected onset temperature and, thus, the

17 larger is the temperature range corresponding to the phase coexistence region as 18 compared to pure DPPC.

19 The order parameter $(S)$ and the rotational diffusion rate $\left(R_{p r p}\right)$, derived from the

20 NLLS simulations, can provide valuable information on the structural arrangements of 21 the bilayer and on the membrane fluidity, respectively. Figure 2 displays the 22 temperature dependence of both parameters simulated with one and two spectral 23 components. In general, the main phase transition is accompanied by abrupt changes in 24 the order parameter (Figure 2A) and the acyl chain mobility (Figure 2B), whereas the 25 pretransition exhibits mostly smooth structural changes of the lipid bilayer as seen by 
1 the paramagnetic end-chain lipid probe. Apart from the increased temperature range of

2 the two-domain coexistence region, the EPR data also show that nicotine has little

3 influence on the main phase transition temperature, confirming our DSC studies.

4 Besides, the drug neither affects the ordering of the bilayer in the gel and fluid phases

5 (Figure 2A) nor the fluidity of the gel phase (Figure 2B). The mobility of the lipids is

6 only changed in the fluid phase at the highest lipid-to-drug molar ratio (10:1). Indeed,

$7 \quad R_{p r p}$ increases by $35-40 \%$ in the fluid phase of DPPC/nicotine membranes as compared

8 to the same phase in the nicotine-free vesicles. This result suggests that nicotine may act

9 as a lipid spacer when adsorbed at high concentrations onto the DPPC surface in the

10 liquid crystalline phase, thus increasing the membrane fluidity of the lipid bilayer.

11 However, nicotine does not alter the activation energy for the 16-PCSL in both gel and

12 fluid phases (Table S1), suggesting that the drug has no effect on the sensitivity to the temperature of the reorientation motion of the molecular axis perpendicular to the long axis of the lipid spin probe in both phases [47]. This result implies that the microviscosity around the nitroxide in the bilayer midplane is virtually the same in the 16 absence and presence of nicotine, yielding similar temperature dependence of the rate of mobility change.

In the phase coexistence region, the major effect of nicotine is a marked increase in the order parameter of the more ordered $L_{\beta^{\prime}}$ phase (Figure 2A). The order parameter of the gel-phase component in the empty DPPC membranes, which is virtually 21 temperature invariant $(S \sim 0.25-0.26)$ over the entire coexistence region, assumes much 22 higher values $(\mathrm{S} \sim 0.3-0.5)$ in the presence of nicotine and becomes linearly dependent on temperature. This result indicates that nicotine induces a membrane structural reorganization by significantly enhancing the packing of the ordered domain in the

25 ripple phase. On the other hand, the ordering of the fluid domain is only slightly 
1 affected by nicotine. We hypothesize that the more packed ordered domain along with

2 the larger temperature range corresponding to the phase coexistence might be the

3 molecular mechanisms behind the enthalpy and entropy gains during phase transition

4 induced by nicotine as compared to the empty membranes, as shown by our DSC

5 experiments. Indeed, more heat is needed to melt more lipids in the $L_{\beta^{\prime}}$ phase and

6 additional entropy is gained in the conformational change between the more ordered,

7 drug-induced, trans-rich lipids to the disordered, gauche-rich lipids.

We can gain further knowledge about the thermodynamics of the DPPC/nicotine system by applying a different theoretical analysis of the data corresponding to the twodomain coexistence region. The population of each spectral component is an important parameter to extract thermodynamical information of the nicotine/lipid system. They can be treated on the basis of a van't Hoff analysis by measuring the equilibrium constant in the phase coexistence region at each temperature. Considering $K$ the equilibrium constant between the lipids in the $L_{\beta^{\prime}}$ and $L_{\alpha}$ domains of the DPPC ripple phase and $K_{P}$ the partition coefficient of 16-PCSL in both domains, then [6]

$$
K(T)=K_{P} \frac{P\left(L_{\alpha}\right)}{P\left(L_{\beta^{\prime}}\right)}=\exp \left(-\frac{\Delta G^{0}}{R T}\right)
$$

where $P\left(L_{\alpha}\right)$ and $P\left(L_{\beta^{\prime}}\right)$ represent the populations of 16-PCSL in the $L_{\alpha}$ and $L_{\beta^{\prime}}$ phases, respectively, obtained by NLLS spectral simulations, $R$ is the universal gas constant, and $\Delta G^{0}$ corresponds to the free energy change necessary to transfer a lipid from the gel state to the fluid state. A plot of $\ln K$ versus $1 / T$ (van't Hoff plot) can thus provide the thermodynamic quantities associated to the phase coexistence region. The van't Hoff analysis has been classified as classical when the plot of $\ln K$ versus $1 / T$ is linear and nonclassical otherwise $[48,49]$. Deviation from linearity has been observed in several 
$1\left(\Delta S^{0}\right)$, and standard heat capacity $\left(\Delta C^{0}\right)$ changes are temperature dependent $[48,49]$. We

2 have previously shown that the dependence of $\ln K$ on $1 / T$ over the temperature range

3 corresponding to the phase coexistence region of DPPC and a pulmonary surfactant

4 lipid model membrane is non-linear and can be written as [6]:

$$
\ln K=\ln \left(\frac{P\left(L_{\alpha}\right)}{P\left(L_{\beta^{\prime}}\right)}\right)=a+\frac{b}{T}+\frac{c}{T^{2}}+\frac{d}{T^{3}}
$$

In the above equation, we assumed that 16-PCSL partitions equally well

6 between the gel and fluid phases of DPPC and is temperature independent, i.e., $K_{P}=1$,

7 as previously reported [15,50-52]. The empirical coefficients $a, b, c$, and $d$ are

8 estimated by fitting Eq. 2 to the experimental $\ln K$ versus $1 / T$ curves and the thermal

9 behavior of the thermodynamic quantities $\left(\Delta G^{0}, \Delta H^{0}, \Delta S^{0}\right.$, and $\left.\Delta C^{0}\right)$ are determined as described elsewhere [6].

Figure 3 illustrates the van't Hoff plots for the 16-PCSL embedded in the membranes studied. The temperature dependence of $\ln K$ obtained for the DPPC membranes was best fitted with a cubic function, as previously observed [6], whereas the effect of nicotine on the gel-fluid equilibrium yielded a parabolic van't Hoff plot. This result is remarkable: qualitatively, binding of nicotine to DPPC membranes affects 16 the van't Hoff behavior of the DPPC/nicotine ripple phase in a way similar to that caused in the $L_{\alpha}-L_{\beta^{\prime}}$ coexistence region of the ternary DPPC/POPC/POPG mixture at 4:3:1 molar ratio due to addition of the unsaturated POPC and POPG lipids to DPPC membranes [6]. Nicotine significantly changed the van't Hoff plots at lower temperatures, indicating different thermal behavior of the spectral populations. Binding of nicotine induces the appearance of a higher population of fluid lipids (50-55\%) as compared to the drug-free membranes $(\sim 30 \%)$ at the onset temperature where phase coexistence is resolved by EPR. Upon heating, the fluid population of the ripple phase 
1 of pure DPPC membranes increases in a non-linear fashion and tends to $50 \%$ at $T_{m}$,

2 whereas molecular rearrangements take place in the DPPC/nicotine ripple phase in such

3 a way that the fluid population decreases to about $37-39 \%$ at $T_{P}$ and then increases to $450 \%$ at $T_{m}$.

The alteration in the non-linear van't Hoff plots of DPPC in the presence of

6 nicotine also leads to different thermotropic behaviors of $\Delta G^{0}, \Delta H^{0}, \Delta S^{0}$, and $\Delta C^{0}$ 7 associated to the equilibrium between the gel and fluid phases, as shown in Figures 4 8 and 5. The best-fit coefficients of the $\ln K$ versus $1 / T$ curves $(a, b, c$, and $d$; Figure 3$)$

9 are shown in the Table S2 of the supplementary material. As can be observed in Figures

$104 \mathrm{~A}$ and $5 \mathrm{~A}$, interconversion of gel-state lipids to fluid-state lipids is more 11 thermodynamically favorable in the presence of nicotine for $T<32-33^{\circ} \mathrm{C}$, i.e., $\Delta G^{0}$ is

12 lower in the presence of the alkaloid and further decreases upon cooling, whereas it 13 increases with lowering $T$ for pure DPPC. On the other hand, for $T>33-34^{\circ} \mathrm{C}$, all $\Delta G^{0}$ 14 curves display similar behavior with temperature and tend to zero as $T$ approaches $T_{m}$. 15 Interestingly, the saddle point of the $\Delta G^{0}$ curve for the empty DPPC membranes (Figure $164 \mathrm{~A}$ ) and the maximum of the curves obtained in the presence of nicotine (Figure 5A) 17 correspond to the pretransition temperature, $T_{P}$, of the membrane systems and can be determined by taking the first derivative of $\Delta G^{0}$ relative to $T$ and setting it to zero. Since $19\left(\partial \Delta G^{0} / \partial T\right)_{P}=-\Delta S^{0}$, the entropy change associated to the isobaric transfer of 1620 PCSL from the solid-ordered gel phase to the disordered fluid phase is zero at $T_{P}$. This 21 result implies that the entropy gained in the conformational change from trans to 22 gauche isomerizations and in the formation of the membrane undulation of the ripple 23 phase is counterbalanced, at $T_{P}$, by the interaction of the head group of the fluid lipids 24 with solvent molecules, which contributes with a negative $\Delta S^{0}$. 
The enthalpy and entropy changes of the two-phase coexistence region are also

2 dramatically modified by nicotine. The $\Delta H^{0}$ and $\Delta S^{0}$ curves obtained for pure DPPC

3 change from a predominantly positive parabolic dependence with the temperature

4 (Figures 4B and 4C) to a linear behavior with $T$ in the presence of nicotine, varying

5 from negative to positive values depending on the temperature (Figures $5 \mathrm{~B}$ and $5 \mathrm{C}$ ).

6 These results are likely due to differences in the heat capacity changes between the gel

7 and fluid domains in the absence and presence of the alkaloid, since $\Delta C^{0}=$

$8\left(\partial \Delta H^{0} / \partial T\right)_{P}=T\left(\partial \Delta S^{0} / \partial T\right)_{P} . \Delta C^{0}$ varies linearly from large negative values, for $T<$

$9 T_{P}$, to large positive values upon raising the temperature for the empty membranes

10 (Figure 4D), whereas it is positive and slightly linearly dependent on $T$ for the nicotine-

11 embedded membranes in the phase coexistence region (Figure 5D). Additionally, $\Delta C^{0}$ is

12 reduced with increased nicotine concentration. Taken together, these findings indicate

13 that nicotine substantially modifies the thermodynamical driving force in the phase

14 coexistence region of DPPC membranes. Indeed, while the thermotropic process

15 associated with the gel-fluid equilibrium (for $T<T_{m}$ ) in pure DPPC is predominantly

16 entropically driven $\left(\Delta H^{0}>0\right.$ and $\left.\Delta S^{0}>0\right)$, nicotine induces an entropically-driven

17 process for $T>T_{P}$ and an enthalpically-driven process for $T<T_{P}$ (Figure 6). Despite

18 that, entropy-enthalpy compensation still holds in the DPPC/nicotine membranes

19 (Figure 6), which indicates that drug binding and membrane perturbation give rise to a

20 very small contribution to the free energy changes between the gel and fluid states, as

21 previously shown for membrane-interacting proteins [53].

At physiological or mildly acidic $\mathrm{pH}$ values, nicotine is a monoprotonated cation

23 with the positive charge centered on the pyrrolidine nitrogen atom [54]. Adsorption of

24 nicotine onto the DPPC membranes should thus be restricted to the membrane/water

25 interface and may likely occur through insertion of the pyridine ring in the bilayer in a 
1 wedge-like conformation, since the pyridine and pyrrolidine rings seem to be oriented

2 roughly perpendicular to one another [55]. The alkaloid does not alter the structure of

3 the DPPC head group and the average hydration property of the lipid bilayer, as

4 demonstrated by the lack of prominent effects on the lipid pretransition (Figure 1A).

5 However, since the positively charged pyrrolidinyl nitrogen may interact

6 electrostatically with the negatively charged phosphate group and with the carboxylate

7 ester groups within the lipid head group, nicotine may compete with water molecules at

8 the membrane surface. Dislocation of water molecules from the lipids may take place

9 but is likely counterbalanced by the nicotine-induced shift of the equilibrium constant

10 towards the formation of fluid lipids, which bind more water molecules than lipids in

11 the gel state [56]. The alkaloid thus changes the hydrogen bonding network in the polar

12 head group region and may stabilize defects at the membrane-water interface [57].

13 These might be the interfacial contributions to the entropy and enthalpy changes

14 between the gel and fluid lipids induced by nicotine. Moreover, the contribution from

15 the acyl chains to both $\Delta H^{0}$ and $\Delta S^{0}$ of the phase coexistence region is two-fold: 1 )

16 negative, due to the substantial ordering effect caused by nicotine in the solid-ordered

17 lipids in the $P_{\beta^{\prime}}$ phase; and 2) positive, due to the higher population of fluid lipids

18 (increased gauche-to-trans conformer ratio) promoted by nicotine as compared to the

19 empty membranes [58]. The ordering effect is likely triggered by electrostatic

20 interactions in the polar region, thus enhancing the van der Waals interactions. As the

21 temperature increases thermal fluctuation gradually decreases the order parameter of the

22 more ordered component in the nicote-containing membranes and changes $K$ so that it

23 modulates the relative contributions of the head group and acyl chains to both $\Delta H^{0}$ and

$24 \Delta S^{0}$ values. 
2 changes the thermotropic behavior of the thermodynamic quantities associated with the

3 two-phase coexistence region in a qualitatively similar way to what was observed in the

4 studies of the ternary 4:3:1 DPPC/POPC/POPG mixture, which mimics the lipid bilayer

5 matrix of the pulmonary surfactant complex [6]. Comparatively to our previous study

6 [6], we found the following from the non-linear van't Hoff analyses: 1) incorporation of

7 nicotine onto pure DPPC substantially modifies the thermodynamic parameters of the

8 two-phase region of the membrane in a way qualitatively similar to the addition

9 unsaturated POPC and POPG lipids into pure DPPC; 2) increasing nicotine

10 concentrations reduced the heat capacity change of the DPPC ripple phase in such a way

11 that resembles the effect of increasing ionic strengths in the DPPC/POPC/POPG

12 mixture; and 3) the positively charged alkaloid seems to have a similar effect in DPPC

membranes as sodium cations in the ternary lipid mixture. Nicotine-induced changes in

14 the van't Hoff plots of DPPC led to marked alterations of the temperature dependence

15 of $\Delta G^{0}, \Delta H^{0}, \Delta S^{0}$, and $\Delta C^{0}$ of the two-phase coexistence region likely due to

16 modifications in the hydrogen-bonding properties and the conformational entropy of the

17 lipids.

In the present work, we extended our previous EPR/van't Hoff analysis of lipid

model membranes exhibiting coexistence of $L_{\beta}-L_{\alpha}$ phases [6] to incorporate the study

of the interaction of a small, drug-like molecule with a two-phase membrane. Increasing evidence shows that the particular composition of biological membranes gives rise to a lateral segregation of their constituents into liquid-disordered and raft-like, liquidordered phases, with the latter being enriched in proteins, cholesterol, and sphingolipids and implicated in a number of important dynamic cellular processes [59]. In particular, the pulmonary surfactant matrix has been shown to display lateral phase separation up 
1 to physiological temperature [60]. Ordered/disordered phase coexistence is thought to

2 be critical for the lung surfactant biophysical function [61]. The interaction of external

3 agents such as drugs, amphiphilis, and/or proteins with surfactant membranes may not

4 only alter the structure and fluidity of the membranes but also disturb the equilibrium

5 between the ordered and disordered domains, potentially leading to complete

6 disappearance of the phase coexistence. Indeed, the surfactant inhibitor C-reactive

7 protein has been shown to bind to surfactant membranes, increase membrane fluidity,

8 and abolish the ordered/disordered phase coexistence, resulting in lung surfactant

9 inactivation [62]. Therefore, we believe that our thermodynamic EPR method might

10 also be useful to shed light on the thermodynamics of complex biological membranes

11 exhibiting lateral phase separation and to investigate how external agents alter the

12 thermodynamical driving force of the phase equilibrium.

\section{Conclusion}

We employed DSC and EPR along with NLLS spectral simulations to study the interaction of nicotine with pure DPPC lipid bilayers. The drug preferentially binds to

17 the fluid phase but does not change the melting profile of the membrane in a prominent

18 way. On the contrary, nicotine slightly increases the enthalpy and entropy changes of 19 the DPPC acyl chain melting transition. NLLS simulations of EPR spectra revelead that 20 the structure, dynamics, and activation energies of the gel and fluid phases are 21 marginally perturbed by the drug. On the other hand, nicotine profoundly alters the van't 22 Hoff behavior of DPPC in the gel-fluid coexistence region. The non-linear, cubic 23 dependence of the free energy change with the temperature is changed to a parabolic 24 behavior in the presence of nicotine. Van't Hoff analysis showed that the thermodynamical driving force and the balance of the non-covalent interactions of the 
1 lipids in equilibrium in the ripple phase of the DPPC bilayer matrix are substantially

2 altered. Transition from a gel state to a fluid state in pure DPPC is predominantly

3 entropically-driven, while nicotine-lipid interactions yield both entropically- and

4 enthalpically-driven processes depending on the temperature. The thermal behavior of

5 the heat capacity change in the phase coexistence region of pure DPPC and

6 DPPC/nicotine lipid bilayers suggested that interaction of nicotine with DPPC alters the

7 relative contributions of the conformational entropy of the lipids and the electrostatic

8 interactions and the $\mathrm{H}$-bond formation in the lipid head group. We believe this

9 thermodynamic EPR approach could be extended to study the interaction of membrane-

10 active molecules with more complex model and biological membranes provided

11 coexistence of different domains could be EPR detected.

13 Acknowledgements

The authors acknowledge the Brazilian agencies FAPESP (Grant nos. 2010/17662-8, 2015/18390-5, 2015/50366-7), CNPq (Grant nos. 475696/2013-1,

16 308380/2013-4) and CAPES for financially supporting this work. LGMB thanks

17 FAPESP for a postdoctoral fellowship (2014/00206-0). The authors are also grateful to

18 the "Sergio Mascarenhas" Molecular Biophysics Group at Sao Carlos Physics Institute

19 of the University of Sao Paulo for allowing access to the DSC and EPR facility. 


\section{REFERENCES}

1 J. M. Boggs, Intermolecular Hydrogen Bonding between Membrane Lipids in: M. Kates, L. A. Manson (Eds.), Membrane fluidity (Biomembranes; v. 12), Plenum Press, New York, 1984, pp. 3-4.

2 McElhaney RN. The use of differential scanning calorimetry and differential thermal analysis in studies of model and biological membranes. Chem Phys Lipids. 1982, 30(2-3):229-259.

3 K. M. W. Keough, P. J. Davis, Thermal Analysis of Membranes, in: M. Kates, L. A. Manson (Eds.), Membrane fluidity (Biomembranes; v. 12), Plenum Press, New York, 1984, pp. 78-73.

4 Heerklotz, H. The microcalorimetry of lipid membranes. J. Phys. Condens. Matter 16 (2004) R441-R467

5 Berliner LJ and Reuben J (1989) Biological magnetic resonance, vol 8: Spin labeling theory and applications (Berliner LJ, Reuben J, eds). Plenum, New York

6 E.D. Vieira, L.G.M. Basso, A.J. Costa-Filho, Non-linear van't Hoff behavior in pulmonary surfactant model membranes, BBA-Biomembranes 1859 (2017) 11331143.

7 Basso, L.G.M., Mendes, L.F.S., Costa-Filho, A.J. The two sides of a lipid-protein story. Biophys Rev (2016) 8: 179-191. https://doi.org/10.1007/s12551-016-0199-5

8 Subczynski WK, Raguz M, Widomska J. Studying lipid organization in biological membranes using liposomes and EPR spin labeling. Methods Mol Biol. 2010, 606:247-69. doi: 10.1007/978-1-60761-447-0_18.

9 Guzzi R, Bartucci R. Electron spin resonance of spin-labeled lipid assemblies and proteins. Arch Biochem Biophys. 2015, 580:102-11. doi: 10.1016/j.abb.2015.06.015.

10 D.E. Budil, S. Lee, S. Saxena, J.H. Freed, Nonlinear-least-squares analysis of slowmotion EPR spectra in one and two dimensions using a modified LevenbergMarquardt algorithm, J. Magn. Reson. Ser. A 120 (1996) 155-189.

11 K.A. Earle, D.E. Budil, Calculating Slow-Motion ESR Spectra of Spin-Labeled Polymers in: Advanced ESR methods in polymer research S. Schlick (Ed.), John Wiley \& Sons, Inc., New Jersey, 2006, pp. 54-83.

12 Costa-Filho AJ, Crepeau RH, Borbat PP, Ge M, Freed JH. Lipid-gramicidin interactions: dynamic structure of the boundary lipid by 2D-ELDOR. Biophys J. 2003, 84(5):3364-78.

13 M.P. Veiga, F.M. Goni, A. Alonso, D. Marsh. Mixed membranes of sphingolipids and glycerolipids as studied by spin-label ESR spectroscopy. A search for domain formation. Biochemistry, 39 (2000), pp. 9876-9883

14 M.T. Ge, A. Gidwani, H.A. Brown, D. Holowka, B. Baird, J.H. Freed. Ordered and disordered phases coexist in plasma membrane vesicles of RBL-2H3 mast cells. An ESR study Biophys. J., 85 (2003), pp. 1278-1288

15 M.J. Swamy, L. Ciani, M.T. Ge, A.K. Smith, D. Holowka, B. Baird, J.H. Freed. Coexisting domains in the plasma membranes of live cells characterized by spinlabel ESR spectroscopy. Biophys. J., 90 (2006), pp. 4452-4465

16 Y. Chiang, A. Costa, B. Baird, J. Freed. 2D-ELDOR study of heterogeneity and domain structure changes in plasma membrane vesicles upon cross-linking of receptors. J. Phys. Chem. B, 115 (2011), pp. 10462-10469

17 Brandts, J.F. The thermodynamics of protein denaturation. I. The denaturation of chymotrypsinogen. J. Am. Chem. Soc. 1964, 86, 4291-4301. 
18 Kao, T.Y.; Tsai, C.J.; Lan, Y.J.; Chiang, Y.W. The role of conformational heterogeneity on regulating the apoptotic activity of BAX protein. Phys. Chem. Chem. Phys. 2017, 19, 9584-9591.

19 Majhi, P.R.; Blume, A. Thermodynamic characterization of temperature-induced micellation and demicellation of detergents studied by differential scanning calorimetry. Langmuir 2001, 17, 3844-3851.

20 Reichenwallner J, Schwieger C, Hinderberger D. Probing the Nanoscopic Thermodynamic Fingerprint of Paramagnetic Ligands Interacting with Amphiphilic Macromolecules. Polymers (Basel). 2017, 9(8). pii: E324. doi: 10.3390/polym9080324.

21 T. Galaon, V. David, Deviation from van't Hoff dependence in RPLC induced by tautomeric interconversion observed for four compounds, J. Sep. Sci. 34 (2011) 1423-1428.

22 S.M. Baumler, A.M. McHale, G.J. Blanchard, Surface charge and overlayer pH influence the dynamics of supported phospholipid films, J Electroanal Chem. 812 (2018) 159-165.

23 E.X. Albuquerque, E.F. R. Pereira, M. Alkondor, S. W. Rogers, Mammalian Nicotinic Acetylcholine Receptors: From Structure to Function, Physiol. Rev. 89 (2009) 73-120.

24 Benowitz NL, Hukkanen J, Jacob P 3rd. Nicotine chemistry, metabolism, kinetics and biomarkers. Handb Exp Pharmacol. 2009, (192):29-60. doi: 10.1007/978-3540-69248-5_2.

25 L.A.J.M. Creuwels, L.M.G. van Golde, H.P. Haagsman. The pulmonary surfactant system: biochemical and clinical aspects. Lung, 175 (1997), pp. 1-39

26 G. van Meer, I. L. van Genderen, Intracellular Lipid Distribution, Transport, and Sorting A Cell Biologist's Need for Physicochemical Information, in: H.J. Hilderson, G.B. Ralston (Eds.), Physicochemical Methods in the Study of Biomembranes (Subcellular Biochemistry; v.23), Plenum Press, New York, 1971, pp. 1-7.

27 L.G.M. Basso, R.Z. Rodrigues, R.M.Z.G. Naal, A.J. Costa, Effects of the antimalarial drug primaquine on the dynamic structure of lipid model membranes , BBA-Biomembranes 1808 (2011) 55-64.

28 R.P. Barroso, L.G.M. Basso, A.J. Costa, Interactions of the antimalarial amodiaquine with lipid model membranes, Chem. Phys. Lipids 186 (2015) 68-78.

29 L.G.M. Basso, E.F.Vicente, E. Crusca Jr., E.M. Cilli A.J. Costa-Filho, SARS-CoV fusion peptides induce membrane surface ordering and curvature, Sci. Rep. 6:37131 (2016) (1-19).

30 Vicente EF, Nobre-Pavinatto TM, Pavinatto FJ, de Oliveira Junior ON, da Costa Filho AJ, Cilli EM. N-Terminal Microdomain Peptide from Human Dihydroorotate Dehydrogenase: Structure and Model Membrane Interactions. Protein Pept Lett. 2015, 22(2):119-29

31 Garcia AF, Simão AM, Bolean M, Hoylaerts MF, Millán JL, Ciancaglini P, CostaFilho AJ. Effects of GPI-anchored TNAP on the dynamic structure of model membranes. Phys Chem Chem Phys. 2015, 17(39):26295-301

32 SG Couto, MC Nonato, AJ Costa-Filho. Defects in Vesicle Core Induced by Escherichia coli Dihydroorotate Dehydrogenase. Biophys J. 2008, 94(5): 17461753. 
33 E. Parra, J. Pérez-Gil, Composition, structure and mechanical properties define performance of pulmonary surfactant membranes and films, Chem. Phys. Lipids 185 (2015) 153-175.

34 K.A. Earle, J. Moscicki, M. Ge, D.E. Budil, J.H. Freed, 250-GHz electron spin resonance studies of polarity gradients along the aliphatic chains in phospholipid membranes, Biophys. J. 66 (1994) 1213-1221.

35 R.N.A.H. Lewis, R.N. McElhaney, The Mesomorphic Phase Behavior of Lipid Bilayers in: P.L. Yeagle (Ed.), The Structure of biological membranes, CRC Press, Florida, 2005, pp. 57-58.

36 R. B Gennis, Biomembranes: molecular structure and function, springer, New York, 1989, pp. 64-60.

37 Janiak MJ, Small DM, Shipley GG. Temperature and compositional dependence of the structure of hydrated dimyristoyl lecithin. J Biol Chem. 1979, 254(13):60686078 .

38 V. Ben Yashar, M. Menashe, R.L. Biltonen, M.L. Johnson, Y. Barenholz. Interaction of trans-parinaric acid with phosphatidylcholine bilayers: comparison with the effect of other fluorophores. Biochimica et Biophysica Acta (BBA) Biomembranes 904 (1987) 117-24.

39 I. Kyrikou, S.K. Hadjikakou, D. Kovala-Demertzi, K. Viras, T. Mavromoustakos. Effects of non-steroid anti-inflammatory drugs in membrane bilayers. Chemistry and Physics of Lipids 132 (2004) 157-169.

40 B.D. Ladbrooke, D. Chapman. Thermal analysis of lipids, proteins and biological membranes. A review and summary of some recent studies. Chem. Phys. Lipids, 3 (1969), pp. 304-356.

41 J.M. Carlson, J.P. Sethna. Theory of the ripple phase in hydrated phospholipid bilayers. Phys. Rev. A, 36 (1987), pp. 3359-3374

42 G. Cevc. Polymorphism of the bilayer membranes in the ordered phase and the molecular origin of the lipid pretransition and rippled lamellae. Biochim. Biophys. Acta, 1062 (1991), pp. 59-69.

43 Y. Lou, M. Ge, J. H. Freed. A Multifrequency ESR Study of the Complex Dynamics of Membranes. J. Phys. Chem. B 2001, 105, 45, 11053-11056.

$44 \mathrm{M}$. Ge and J. H. Freed. Hydration, Structure, and Molecular Interactions in the Headgroup Region of Dioleoylphosphatidylcholine Bilayers: An Electron Spin Resonance Study. Biophys J. 2003, 85(6):4023-40.

45 K. Sengupta, V.A. Raghunathan, J. Katsaras Structure of the ripple phase of phospholipid multibilayers. Phys. Rev. E, 68 (2003), pp. 1-12

46 R.J. Wittebort, A. Blume, T.H. Huang, S.K. DasGupta, R.G. Griffin. Carbon-13 nuclear magnetic resonance investigations of phase transitions and phase equilibria in pure and mixed phospholipid bilayers. Biochemistry, 21 (1982), pp. 3487-3502

47 K. Nakagawa, Diffusion Coefficient and Relaxation Time of Aliphatic Spin Probes in a Unique Triglyceride Membrane, Langmuir 19 (2003) 5078-5082.

48 D. Haidacher, A. Vailaya, C. Horvath, Temperature effects in hydrophobic interaction chromatography, Proc. Natl. Acad. Sci. U. S. A. 93 (1996) 2290-2295.

49 R.I. Boysen, Y. Wang, H.H. Keah, M.T.W. Hearn, Observations on the origin of the non-linear van't Hoff behaviour of polypeptides in hydrophobic environments, Biophys. Chem. 77 (1999) 79-97.

50 I. Ionova, V. Livshits, D. Marsh. Phase diagram of ternary cholesterol/palmitoylsphingomyelin/palmitoyloleoyl-phosphatidylcholine mixtures: 
spin-label EPR study of lipid-raft formation. Biophys. J., 102 (2012), pp. 18561865

51 Y. Chiang, Y. Shimoyama, G. Feigenson, J.H. Freed, Dynamic molecular structure of DPPC-DLPC-cholesterol ternary lipid system by spin-label electron spin resonance, Biophys. J. 87 (2004) 2483-2496.

52 Y.W. Chiang, J. Zhao, J. Wu, Y.H. Shimoyama, J.H. Freed, G.W. Feigenson, New method for determining tie-lines in coexisting membrane phases using spin-label ESR , BBA-Biomembranes 1668 (2005) 99-105.

53 Jahnig, F. Thermodynamics and kinetics of protein incorporation into membranes. Proc. Natl. Acad. Sci. U. S. A. Biol. Sci., 80 (1983), pp. 3691-3695

54 P.M. Clayton, C.A. Vas, T.T.T. Bui, A.F. Drake, K. McAdam, Spectroscopic investigations into the acid-base properties of nicotine at different temperatures, Anal. Methods 5 (2013) 81-88.

55 J. Graton, T. van Mourik, S. L. Price, Interference between the hydrogen bonds to the ringue of nicotine, J. Am. Chem. Soc. 125 (2003) 5988-5997

56 Tristram-Nagle S. (2015) Use of X-Ray and Neutron Scattering Methods with Volume Measurements to Determine Lipid Bilayer Structure and Number of Water Molecules/Lipid. In: Disalvo E. (eds) Membrane Hydration, 17-43. Subcellular Biochemistry, vol 71. Springer, Cham

57 E.A. Disalvo, F. Lairion, F. Martini, E. Tymczyszyn, M. Frías, H. Almaleck, G.J. Gordillo, Structural and functional properties of hydration and confined water in membrane interfaces, BBA-Biomembranes 1778 (2008) 2655-2670.

58 T. Heimburg. Lipid Melting. Wiley-VCH Verlag GmbH \& Co. KGaA (2007)

59 Lingwood D, Simons K. Lipid rafts as a membrane-organizing principle. Science. 2010, 327(5961):46-50. doi: 10.1126/science.1174621.

60 J. Bernardino de la Serna, J. Perez-Gil, A.C. Simonsen, L.A. Bagatolli. Cholesterol rules: direct observation of the coexistence of two fluid phases in native pulmonary surfactant membranes at physiological temperatures. J. Biol. Chem., 279 (2004), pp. 40715-40722

61 Casals C, Cañadas O. Role of lipid ordered/disordered phase coexistence in pulmonary surfactant function. Biochim Biophys Acta. 2012, 1818(11):2550-62. doi: 10.1016/j.bbamem.2012.05.024.

62 A. Sáenz, A. López-Sánchez, J. Mojica-Lázaro, L. Marínez-Caro, N. Nin, L.A. Bagatolli, C. Casals. Fluidizing effects of C-reactive protein on lung surfactant membranes: protective role of surfactant protein A. FASEB J., 24 (2010), pp. 36623673 


\section{Table caption}

Table 1. Calorimetric parameters obtained from the DSC thermogram of nicotine-free and nicotine-containing DPPC lipid vesicles at different lipid-to-drug molar ratios. $\Delta H_{\text {cal }}$ represents the enthalpy change of the whole phase transition curve; $T_{m}$ is the main phase transition temperature; $T_{p}$, the pretransition temperature; $\Delta T_{m, 1 / 2}$, the width at half height of the main phase transition peak; $\Delta S$ represents the entropy change of the transition at $T_{m} ; \Delta H_{v H}$, the van't Hoff enthalpy; and CUS, the cooperative unit size, given in number of molecules.

\section{Figures captions}

Figure 1. Thermotropic phase behavior of the membranes as monitored by DSC. (A) Temperature dependence of the molar heat capacity of DPPC in the absence and presence of nicotine at different lipid-to-drug molar ratios. The inset illustrates the pretransition of DPPC. (B) Effect of nicotine on the enthalpy change ( $\Delta H$, circles) and the melting temperature ( $T_{m}$, triangles) of DPPC lipid vesicles.

Figure 2. Thermotropic phase behavior of the membranes as monitored by EPR. Temperature dependence of (A) the order parameter $S$ and (B) the rotational diffusion rate $R_{p r p}$ of 16-PCSL in pure DPPC (top panels) and DPPC/nicotine lipid vesicles at two lipid-to-drug molar ratios: 25:1 (middle panels) and 10:1 (bottom panels). Both parameters were obtained from the NLLS simulations of the EPR spectra using one (full black squares) or two (open squares and circles) spectral components.

Figure 3. Van't Hoff analysis of pure DPPC and DPPC/nicotine multilamellar vesicles. The solid lines are the best fits to the van't Hoff plots. Pure DPPC was best fitted with a cubic function, whereas the effect of nicotine yielded a parabolic van't Hoff plot. The lipid-to-drug molar ratios were 25:1 and 10:1.

Figure 4. Thermodynamics of the phase coexistence region of DPPC vesicles. Temperature-dependence of the changes in the (A) Gibbs free energy, $\Delta G^{0}$, (B) enthalpy, $\Delta H^{0},(\mathbf{C})$ entropy, $\Delta S^{0}$, and (D) heat capacity, $\Delta C^{0}$.

Figure 5. Thermodynamics of the phase coexistence region of DPPC/nicotine vesicles. Temperature-dependence of the changes in the (A) Gibbs free energy, $\Delta G^{0},(\mathbf{B})$ enthalpy, $\Delta H^{0},(\mathbf{C})$ entropy, $\Delta S^{0}$, and (D) heat capacity, $\Delta C^{0}$ for nicotine-containing membranes at lipid-to-drug molar ratios of 25:1 (solid squares) and 10:1 (open circles).

Figure 6. Entropy-enthalpy compensation of the DPPC/nicotine vesicles. $T \Delta S^{0} \mathrm{x}$ $\Delta H^{0}$ plots for pure DPPC (solid squares) and DPPC/nicotine vesicles at lipid-to-drug molar ratios of 25:1 (open circles) and 10:1 (open triangles). 


\section{Table 1}

\begin{tabular}{ccccccccc}
\hline Sample & $\begin{array}{c}\Delta H_{c a l}{ }^{*} \\
(\mathbf{k c a l} / \mathbf{m o l})\end{array}$ & $\begin{array}{c}T_{m} \\
\left({ }^{\circ} \mathbf{C}\right)\end{array}$ & $\begin{array}{c}\Delta T_{m, 1 / 2} \\
\left({ }^{\circ} \mathbf{C}\right)\end{array}$ & $\begin{array}{c}T_{p} \\
\left({ }^{\circ} \mathbf{C}\right)\end{array}$ & $\begin{array}{c}\Delta S^{*} \\
(\mathbf{c a l} / \mathbf{m o l} / \mathbf{K})\end{array}$ & $\begin{array}{c}\Delta H_{v H}{ }^{*} \\
(\mathbf{k c a l} / \mathbf{m o l})\end{array}$ & CUS $^{*}$ \\
\hline blank & 8.74 & 41.00 & 0.14 & 34.0 & 27.8 & 2,930 & 335 \\
$50: 1$ & 9.28 & 40.97 & 0.13 & 34.0 & 29.5 & 2,990 & 322 \\
$25: 1$ & 9.52 & 40.94 & 0.13 & 33.9 & 30.3 & 2,980 & 312 \\
$10: 1$ & 9.64 & 40.86 & 0.12 & 33.6 & 30.7 & 3,250 & 337 \\
$5: 1$ & 9.99 & 40.73 & 0.13 & 33.1 & 31.8 & 2,990 & 299 \\
$2: 1$ & 9.96 & 40.34 & 0.13 & 31.6 & 31.8 & 2,740 & 275 \\
\hline
\end{tabular}

Estimated errors: $\Delta H_{\text {cal }}(0.05 \mathrm{kcal} / \mathrm{mol}), T_{m}\left(0.02^{\circ} \mathrm{C}\right), \Delta T_{m, 1 / 2}\left(0.03^{\circ} \mathrm{C}\right), T_{p}\left(0.1^{\circ} \mathrm{C}\right), \Delta S(0.2 \mathrm{cal} / \mathrm{mol} / \mathrm{K})$, $\Delta H_{v H}(20 \mathrm{kcal} / \mathrm{mol})$.

${ }^{*} \Delta H_{\text {cal }}$ was calculated from the area under the $C_{P}$ curve, $\Delta S=\Delta H_{\text {cal }} / T_{m}, \Delta H_{v H}=4 R T_{m}^{2} C_{p, \max } / \Delta H_{\text {cal }}$, and CUS $=\Delta H_{v H} / \Delta H_{\text {cal }}$. 


\section{Figure 1}

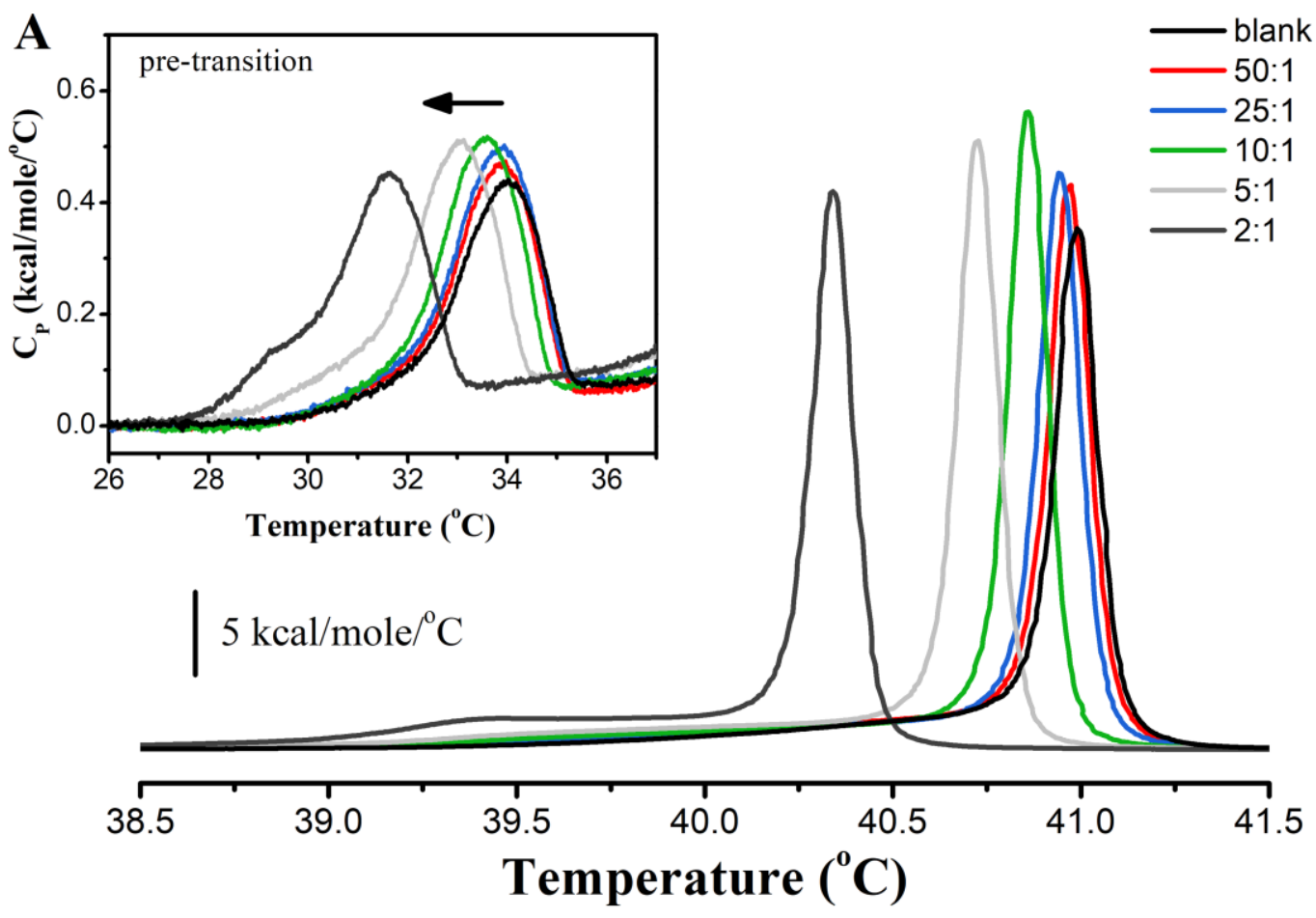

B

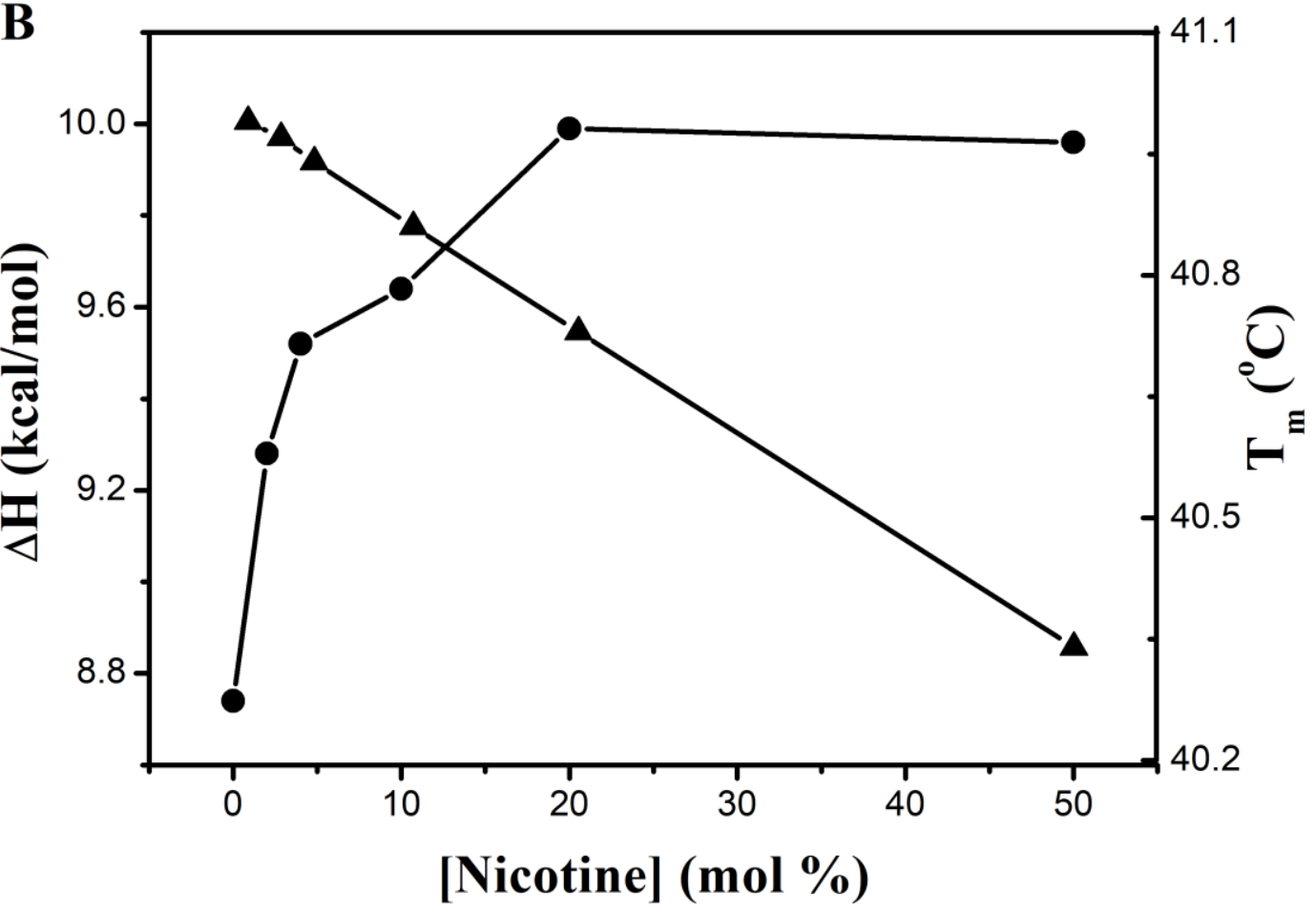




\section{Figure 2}
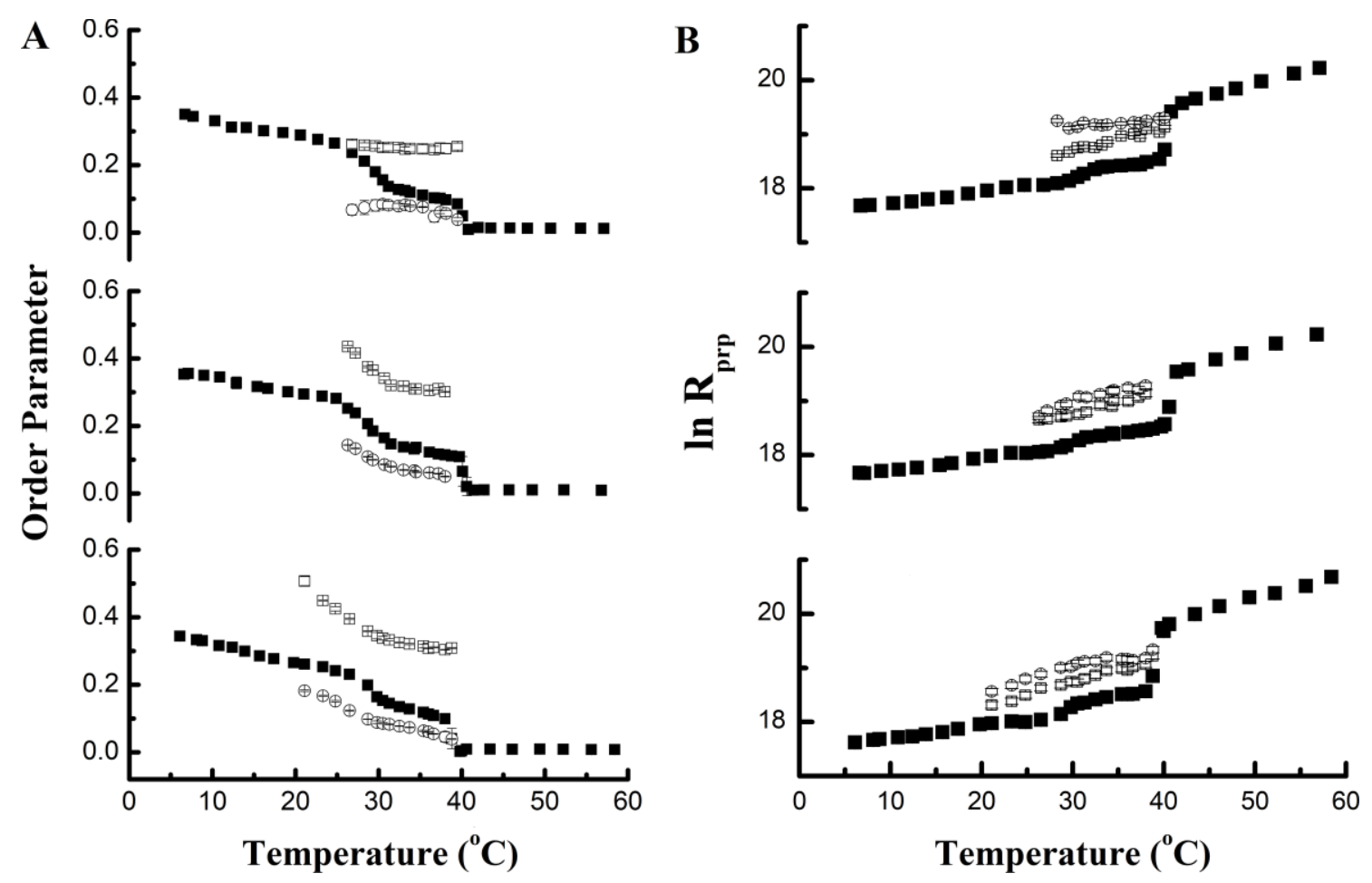


\section{Figure 3}

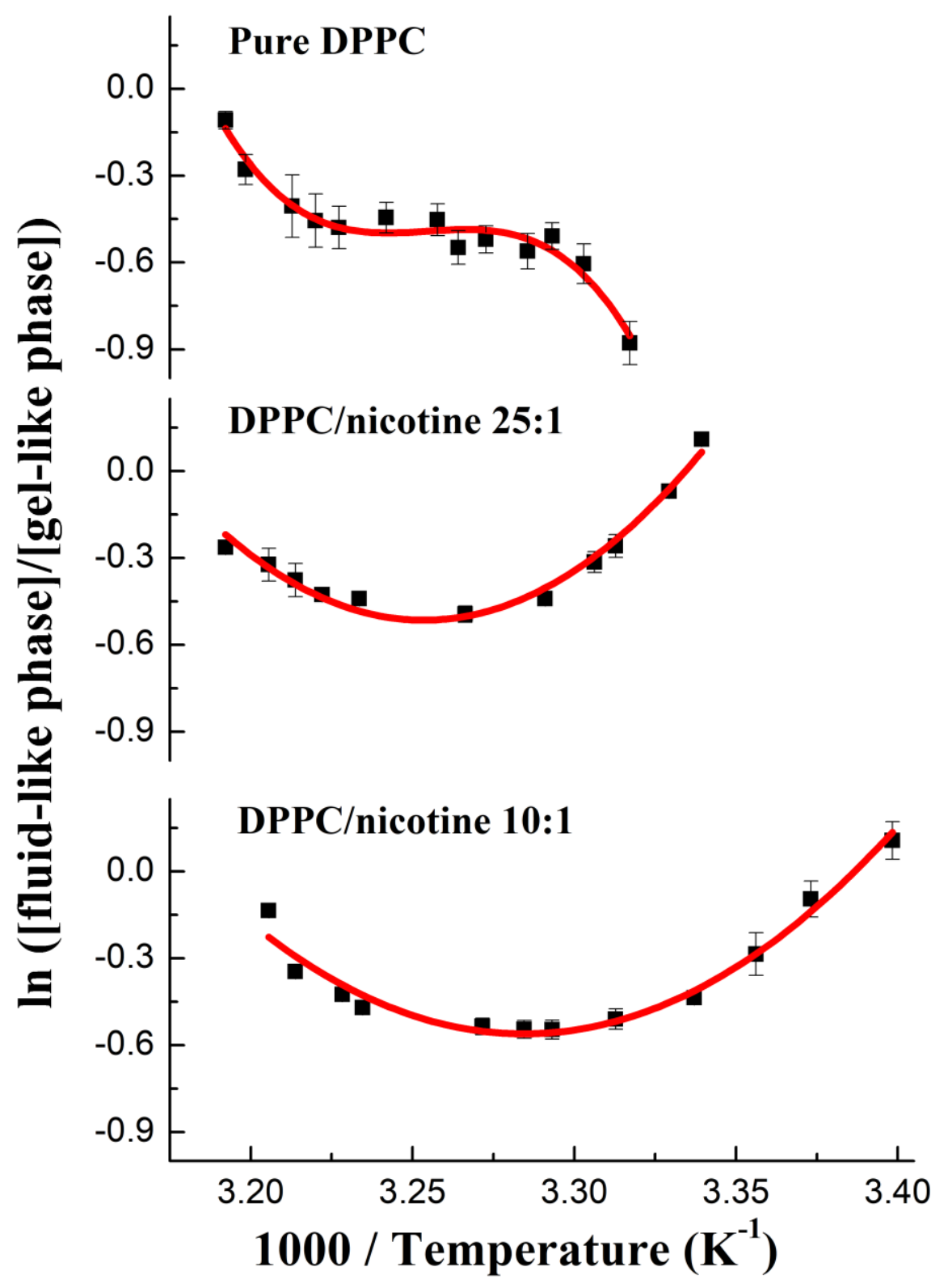


Figure 4
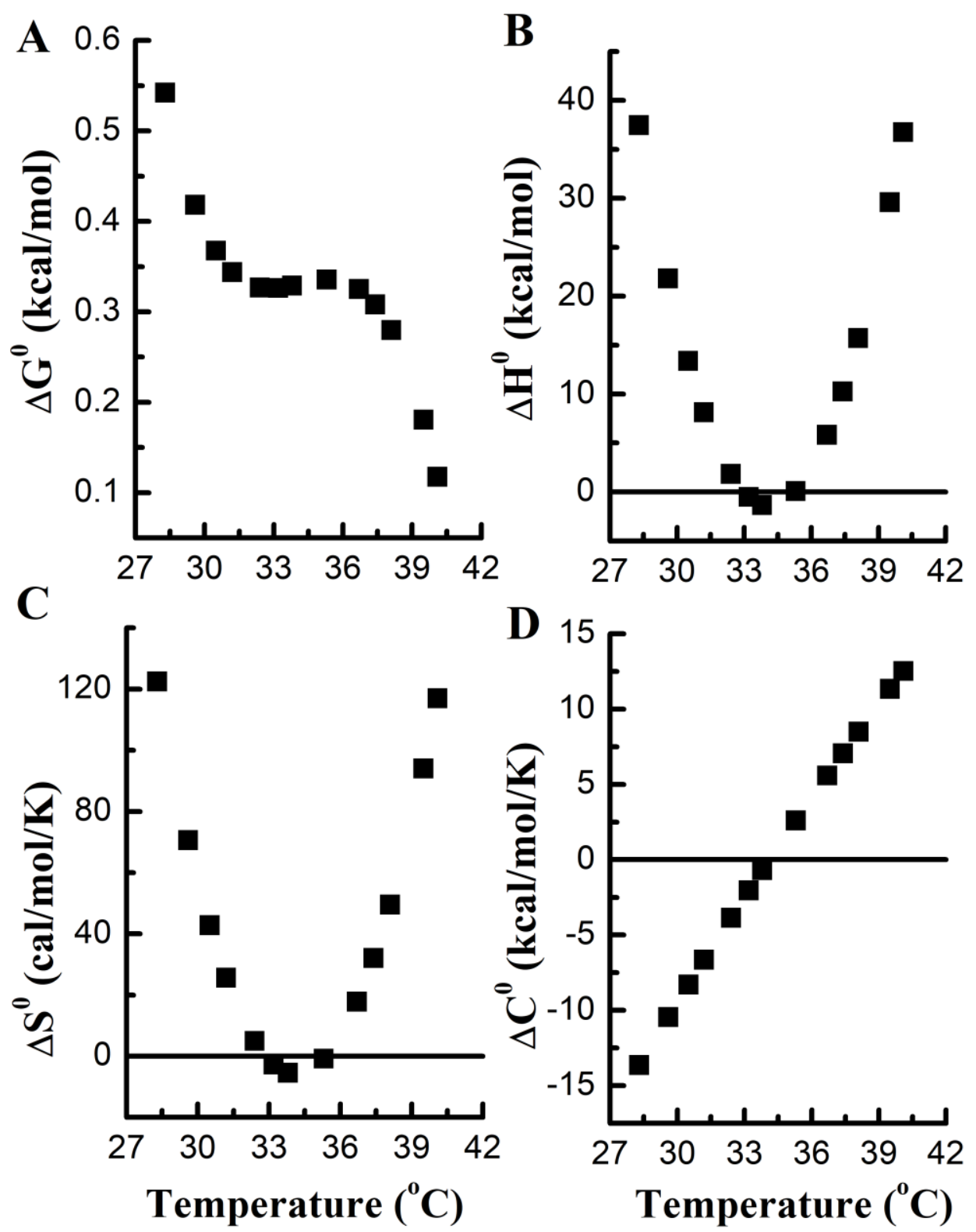
Figure 5
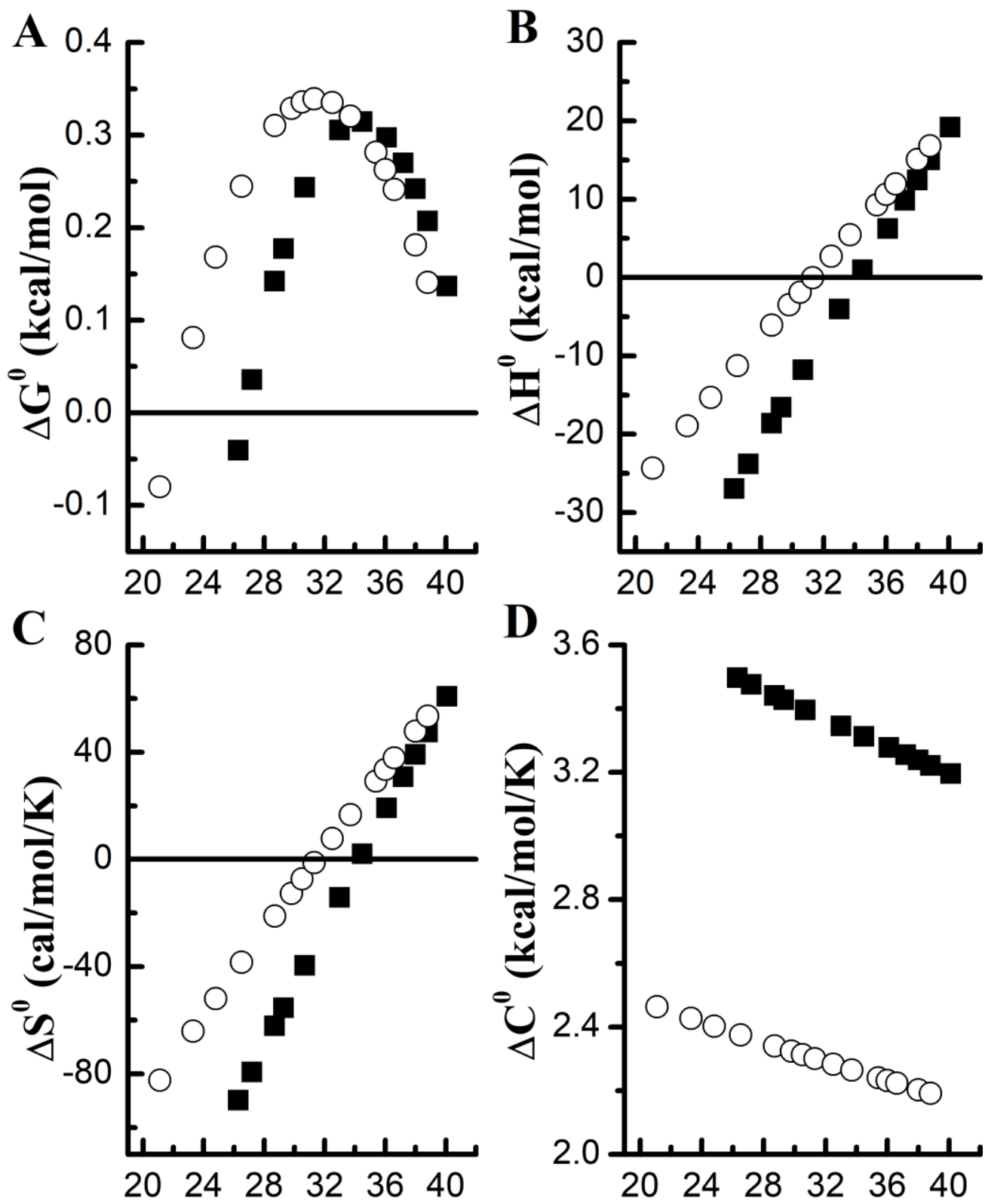

D

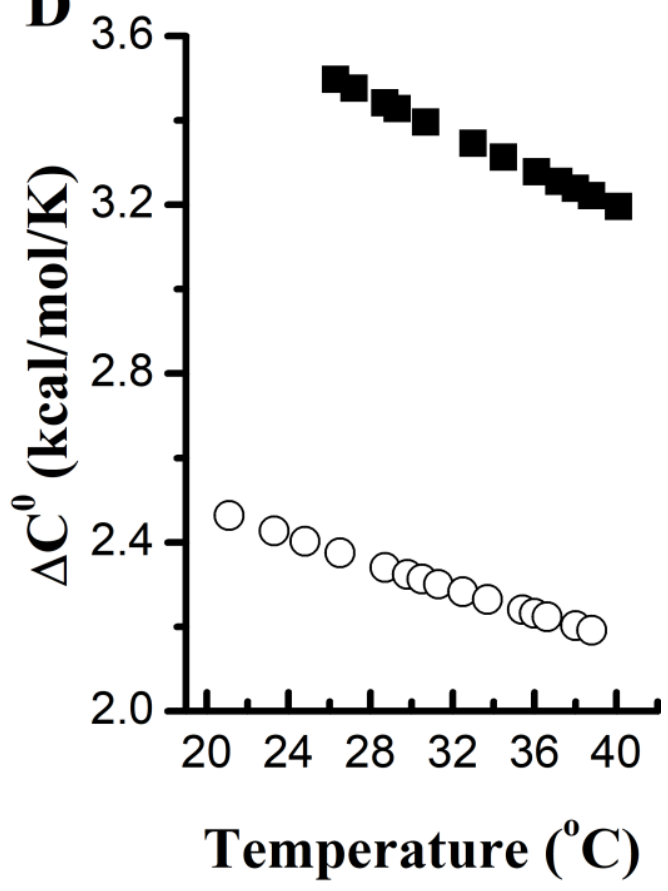

Temperature $\left({ }^{\circ} \mathrm{C}\right)$ 
Figure 6

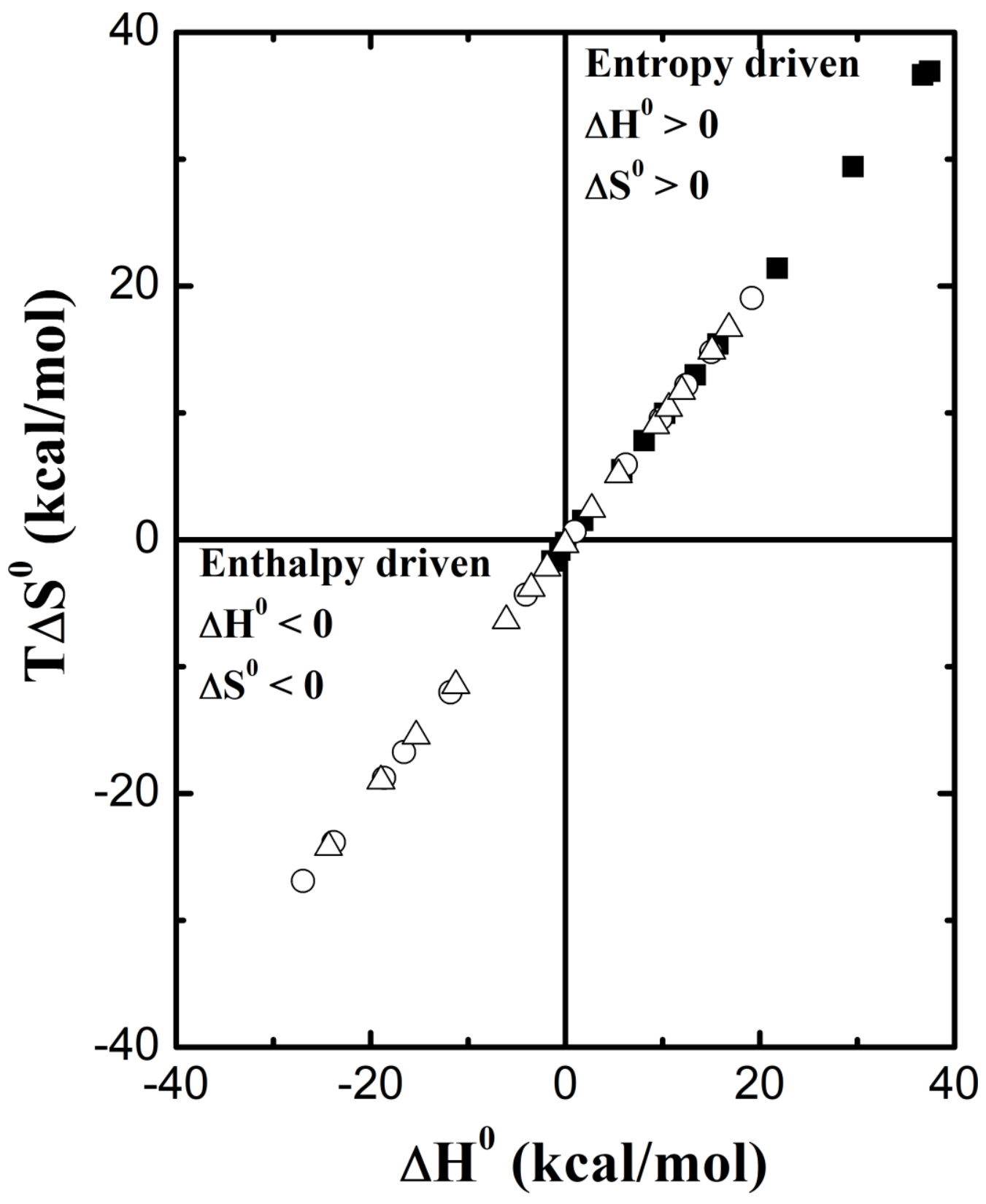

